\title{
7. Symmetric Space-time
}

I have argued that unless the aether is discrete we should adopt a revisionary interpretation of General Relativity, according to which Spacetime is not lumpy and bumpy like a potato, but is affine or de Sitterish, or of some other nice symmetric shape.

Is the aether itself symmetric? From its symmetry it would follow that the it pervades the whole of Space-time. On the Arntzenius Continuum hypothesis, they would differ only in that Space-time is made up of point locations, constructed from regions in the way described in Chapter Four. This would exclude both the Growing Block and Presentism, if these are interpreted as denying the reality of any future aether. For if there is no future aether then the aether is not invariant under those symmetries that map the past to the future.

There is another complication with the use of symmetries to characterize the aether. Realists about universals have at their disposal a group-theoretic approach based on realism about symmetries, which are dyadic relations between regions of aether. Nominalists might, however, reject symmetries, in which case they are committed to an alternative way of characterizing symmetric Space-time, using axioms. This is fairly straightforward for affine and Minkowski Space-time, but for other symmetric spaces such as de Sitter Space-time I do not know of axiomatic treatments.

One final issue concerns the characterization of Space-time if the aether is point-free. The axiomatic approach requires quite a serious complication; the group-theoretic one generalizes straightforwardly.

I shall begin by considering the axiomatic approach. I then suppose the aether is point-based and consider the group-theoretic approach for flat Space-time, before generalizing it to the point-based curved case, and the point-free cases. 


\section{The axiomatic approach}

\section{The point-based case}

There are intuitive axioms (Coppel 1998) that ensure a point-based continuous aether is representable as flat. The primitive relation used is that of metathety, namely the relation that holds between points $\mathrm{p}, \mathrm{q}$ and $\mathrm{r}$ in that order if they lie on a straight line and $q$ is between $p$ and $r$.

The requirement for a mapping $\mathrm{F}$ from the points to the quadruples to be a (faithful) representation is that:

$\mathrm{F}$ is a 1 to 1 mapping and; if $\mathrm{q}$ is between $\mathrm{p}$ and $\mathrm{r}$, then $\mathrm{F}(\mathrm{q})$ is a convex linear combination of $F(p)$ and $F(r)$, that is, for some real number $\mathrm{x}, 0 \leq \mathrm{x} \leq 1$, and $\mathrm{F}(\mathrm{q})=\mathrm{xF}(\mathrm{p})+(1-\mathrm{x}) \mathrm{F}(\mathrm{r})$.

Coppel's axioms are sufficient to ensure that the aether is representable as flat. Moreover, if the convex region in question is not the set of all quadruples of real numbers, then we may take Space-time point locations to correspond to all the quadruples, exhibiting the aether as occupying a convex portion of Space-time, which would be sufficient to accommodate the Growing Block or Presentism. (Here I am supposing the presentist believes in a present layer of the aether of, say, a Planck time thickness.)

Being representable as flat is not the same as being flat, however. So this approach, although basically sound, must be modified to include hypervolume (or, more austerely, the comparative quantity relation) as primitive in addition to metathety. Moreover, even if the aether is flat the representation could be misleading, because of fine structure in the form of extra dimensions that do not contribute to the quantity. Compare aether of three spatial and one temporal dimension, with a merely possible aether in which only the temporal and two of the three spatial dimensions contribute to the quantity. Ignoring Time for the sake of simplicity this would be a two dimensional aether except that it has an extra infinitesimal thickness in the third dimension. In both the actual and the merely possible cases, the axioms concerning metathety would be satisfied, and the number of dimensions characterized as three. But I consider that the difference between the two cases should be reflected in the axioms, and this can be done, in 3 dimensions, as follows. In the actual case, but not the other one, if we take any 4 points none of which 
is part of every convex region containing the other 3 , then any convex region containing all 4 of them must have positive quantity.

A more serious misrepresentation of the aether would occur if in fact it is curved but conformal to flat. Consider Einsteinian manifolds, that is, 4-dimensional manifolds with a general relativistic 'metric'. David Malament's result (1977) shows that their structure is specified by the light cones and the hypervolume, but it is not specified by the light cones alone. In particular, there can be an Einsteinian manifold E that is not flat and yet there is a 1 to 1 onto mapping $\mathrm{H}$ from $\mathrm{E}$ to a Minkowski Space-time, $\mathrm{M}$, such that $\mathrm{H}$ and its inverse are smooth (infinitely differentiable) and that $\mathrm{H}$ maps light cones to light cones and geodesics to geodesics. The mapping $\mathrm{H}$ also preserves metathety, if we define it replacing 'straight line' by 'geodesic'. Hence E satisfies the axioms for a flat Space-time. To avoid treating $\mathrm{E}$ as flat we need to distinguish $\mathrm{E}$ from $\mathrm{M}$ using the hypervolume.

The easiest way of introducing the hypervolume to solve this problem is requiring it to be translation-invariant, which is an immediate consequence of the symmetry-theoretic approach. This is not so straightforward using axioms, however. We may characterize parallelepipeds and then consider one of them, $u$, divided into two of equal hypervolume, $\mathrm{v}$ and $\mathrm{w}$ by a hyperplane parallel to one of $\mathrm{u}$ 's faces, and divided into another two of equal hypervolume, $\mathrm{x}$ and $\mathrm{y}$ by a hyperplane parallel to another of u's faces. The requirement that the four parallelepipeds $\mathrm{v} \wedge \mathrm{x}, \mathrm{v} \wedge \mathrm{y}, \mathrm{w} \wedge \mathrm{x}$, and $\mathrm{w} \wedge \mathrm{y}$ are always of equal hypervolume should, I think, ensure the translation invariance of hypervolume.(See Diagram Two for the two dimensional analog.) 


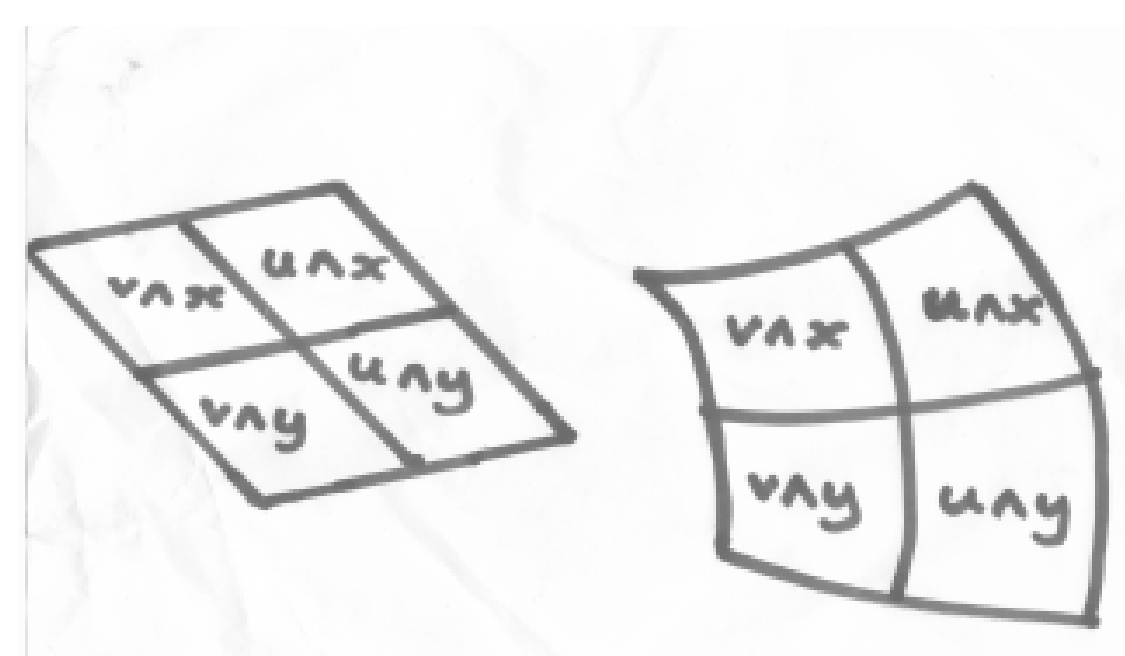

Diagram Two

I conclude that the axiomatic characterization of affine, or, more generally, flat aether succeeds in the point-based case, but at the cost of some complexity. In Chapter Five I explained why if we treat gravity as just another field we can, in the case of a flat Space-time, choose between a Minkowski structure or the, more general, affine structure. ${ }^{146}$ Economy supports the latter, but I note that, as Alfred Robb (1914) showed, we can find axioms that characterize Minkowski Space-time. ${ }^{147}$

146 Bearing in mind that a flat Space-time is affine only if it extends infinitely in all dimensions. For ease of exposition I am here ignoring spatially finite flat Space-times.

Robb was the pioneer. His treatment is not merely rather complicated but it characterises as Minkowskian, Space-times that are merely conformably equivalent to Minkowskian. This is the problem that I considered above when discussing affine aether. In addition Robb assumes there are points.

I recommend an axiomatic system that takes the part/whole relation, frame-independent temporal priority, and comparative quantity as basic. Using these, we may characterise a metathety relation between past light cones that corresponds in the point-based case to metathety of the vertices of the cones. Then we use the axioms for affine spaces stated in terms of the metathety and hypervolume, mentioned above. Finally we require some constraint on the past light cones to ensure they have the right shape to be cones. This last step is quite easy, because we can characterise a two dimensional slice of 


\section{The point-free case}

Even if the aether itself is point-free, I take Space-time to be made of points (point locations). A region of Space-time $w$ is said to be convex if whenever two points $\mathrm{p}$ and $\mathrm{r}$ belong to $\mathrm{w}$ any point $\mathrm{q}$ between $\mathrm{p}$ and $\mathrm{r}$ also belongs to $\mathrm{w}$. This idea of convexity is, I suggest, a natural concept and in the point-free case we may take it as a primitive property that some regions of the aether have and others lack. We may use it to provide an axiomatic characterization of point-free affine aether, without any mention of the constructed points.

We may then say that an aether region $u$ has flat boundary if both $\mathrm{u}$ and some complement of $\mathrm{u}$ are convex. In the case of greatest interest, Arntzenius Continuum, every region has a unique complement so we may say a region has flat boundary if both it and its complement are convex. I shall restrict attention to that case.

The unordered pair $\{\mathrm{u}, \mathrm{v}\}$ consisting of two convex regions each the complement of the other is a surrogate for a hyperplane, so in this case I denote $\{\mathrm{u}, \mathrm{v}\}$ as $\mathrm{H}(\mathrm{u}, \mathrm{v})$. We expect both $\mathrm{u}$ and $\mathrm{v}$ to be representable by open sets of quadruples separated by a hyperplanar set of quadruples.

Given two distinct hyperplane-surrogates $\mathrm{H}(\mathrm{x}, \mathrm{y})$ and $\mathrm{H}(\mathrm{u}, \mathrm{v})$ it might happen that one of $\mathrm{u}, \mathrm{v}, \mathrm{x}$ or $\mathrm{y}$ has another of $\mathrm{u}, \mathrm{v}, \mathrm{x}$ or $\mathrm{y}$ as a proper part. In the Arntzenius Continuum case we expect this to occur only if the two representing hyperplanar sets fail to intersect. We may therefore use this to characterize the relation of being parallel, where I stipulate that being parallel is anti-reflexive. It will then be an axiom that the relation of being either identical or parallel is transitive. From its definition it is symmetric so it is an equivalence relation. An axiomatic

a cone in $\mathrm{N}$ dimensions using the converse of Pascal's theorem for conic sections.

The first step can be broken down into two stages. First we may characterise metathety for 'points' (i.e. vertices of cones) that are neither space-like nor time-like separated but lie on a light ray. For a past light cone and a future light cone that touch but have no overlap of positive volume must define such a line. Then we use Pappus' Theorem to characterise other cases of metathety. 
characterization of $n$ dimensional affine aether, for $n \geq 3$, may then be obtained by:

1. Requiring the equivalence classes of hyperplane-surrogates to satisfy the axioms for the (dual of) a projective space of $n-1$ dimensions over the real numbers; and

2. Putting constraints on the hypervolume similar to those for the point-based case.

If we construct the points of Space-time using the ultrafilter construction we will thus obtain an affine structure for the Space-time in which the regions are located. This requires, however, the aether to occupy all of Space-time, whereas in the point-based case it sufficed that the aether occupied only a convex region. (We need all Space-time to ensure that the relation that we treat as being parallel is a genuine equivalence relation.) So this is not compatible with the strict presentist or Growing Block theories that deny the existence of future fundamental things such as future portions of the aether. To get around this restriction we could consider the one-cornered regions that are meets of 4 suitably arranged regions with flat boundaries. Such regions act as surrogate points, and we may characterize when three of them are lined up in such a way that the corner of one of them is between the other two corners and on a straight line. But that is a messy way of characterizing affine aether.

\section{The group-theoretic characterisation of affine aether}

Inspired by Felix Klein's Erlanger program, in which different geometries are characterised algebraically by their structure-preserving mappings, I shall show how in some cases the geometry of the aether may be characterised using a group of symmetries. I begin with the most straightforward cases, those in which the aether is point based and pervades the whole of Space-time, with which it may be identified. In this case the symmetries are global. Later I shall consider local symmetries.

A symmetry $\mathrm{g}$ is here taken to be a universal. As mentioned in the Introduction this supposition requires quasi-realism about universals, namely either realism or a nominalist theory that can mimic realism. If I want to stress that a symmetry $g$ is a relation I write it as Rg. Because $g$ maps regions of aether in a one to one onto fashion, if Rguv and Rgwx, 
then $\mathrm{u}=\mathrm{w}$ if and only if $\mathrm{v}=\mathrm{x}$. As a symmetry, $\mathrm{g}$ preserves the fundamental properties of and relations between points. In the affine case I shall characterise the aether using four primitives: the part/whole relation of mereology, the comparative quantity relation, the property of convexity, and the idea of a symmetry itself. So I require that the symmetry preserve symmetries. This follows from the assumption that for any symmetry $\mathrm{g}$, and any region $\mathrm{x}$ there are regions $\mathrm{y}$ and $\mathrm{z}$ such that Rgxy and Rgzx. In that case, given the convention that identity is a symmetry, the symmetries form a group. That is, any two symmetries have a composite that is also a symmetry, composition is associative and any symmetry $g$ has an inverse, $g^{-1}$, which when composed with $g$ results in the identity map. The symmetry $g$ then preserves symmetry in the sense that if $h$ is any symmetry $g-1 \circ h \circ g$ is also a symmetry. The topology is derived from convexity and hypervolume so the relation of separation is also preserved. ${ }^{148}$

The inclusion of the concept of symmetry among the primitives is required, I hold, if we are to use symmetry to describe the nature of the aether. This is a problem because symmetry supervenes upon the other primitives, and so it would seem more economic to take it to depend ontologically on the other primitives. This is a known problem for those who take both universals and particulars to be fundamental. For instance, the existence of the universal being spherical supervenes on the spherical objects but neither the objects nor the universal are thought of as less fundamental than the others. One sort of nominalist seeks to solve the problem by taking the universal to depend for its existence on the particulars and their resemblance. On the other hand, a bundle theorist solves the problem by treating the particulars as just bundles of universals. My own preferred solution is platonist - the universals are necessary beings and so supervene on the particulars only in a trivial sense that does not

We may characterise the (not necessarily open) regions with flat boundary as those convex regions with convex complement. Two convex regions $\mathrm{u}$ and $\mathrm{v}$ are separated if they are parts of disjoint regions with flat boundary $\mathrm{w}$ and $\mathrm{x}$ such that the meet of the complements, $\neg \mathrm{W} \wedge \neg \mathrm{x}$ is of positive hypervolume. Then two regions $y$ and $z$ are separated if every convex part of $y$ is separated from every convex part of $\mathrm{z}$. 
threaten their being taken as primitives. In any case, whatever solution we adopt generally for universals applies to the case of symmetries.

Initially I shall assume that no two symmetries are actually coextensive: that is if $g(u)=h(u)$ for every region $u$ then $g=h$. If this holds then I say that the group of symmetries acts faithfully. In the next section I relax that requirement.

The group of symmetries $\mathrm{G}$ inherits a topological structure from the aether. Given any regions $\mathrm{u}$ and $\mathrm{v}$, the set $\mathrm{U}(\mathrm{u}, \mathrm{v})=\{\mathrm{g} \in \mathrm{G}: \mathrm{g}(\mathrm{u}) \ll \mathrm{v}\}$ must be open, although it might well be $\varnothing$, and the open sets of symmetries are precisely the unions of some of the $U(u, v)$. The theory of topological groups is well developed and, as noted in Chapter Five, under fairly weak constraints $\mathrm{G}$ has a unique structure as a Lie group, that is, a group with a natural differentiable manifold structure. Not only does this solve the problem of characterising differentiable structure, but it also enables us to use the classification of Lie groups to describe hypothetical geometric structures for the aether.

In the point-based case, affine aether may be characterised by requiring the following:

1. The group of all symmetries $\mathrm{G}$ has just one transitive commutative sub-group of symmetries, $\mathrm{V}$, which we call the vectors. (To say that $\mathrm{V}$ is transitive is to say that given any two point parts $\mathrm{u}$ and $\mathrm{v}$ of the aether there is some $h \in V$ such that $h(u)=v$. $)^{149}$

2. V has no compact subgroup except the trivial one $\{\mathrm{Id}\}$.

3. $\mathrm{V}$ is connected and locally compact. We may then treat $\mathrm{V}$ as a real vector space, writing gok as $\mathrm{g}+\mathrm{k}$, Id as 0 , and the inverse $\mathrm{g}^{-1}$ of $\mathrm{g}$ as $-\mathrm{g}$. Then the number of dimensions, $\mathrm{M}$, is the

149 (1) implies that $G$ acts faithfully. This is relevant, because it is conceivable that there be vectors and schwectors that are co-extensive. In that case the group of vectors could not be characterised as the only transitive faithful subgroup of all the symmetries.

Conditions (2) and (3) imply that V is a connected Lie group. All commutative connected Lie groups of dimension $\mathrm{M}$ have the same Lie algebra (with $[\mathrm{x}, \mathrm{y}]=0$ for all $\mathrm{x}$ and $\mathrm{y}$.) The one dimensional subgroups must be either circles or lines. Circles are excluded by (2), so V must be a real vector space of M dimensions. 
smallest cardinality of any subset $\mathrm{X}$ of $\mathrm{V}$ that generates $\mathrm{V}$. That is, $\mathrm{V}$ is the smallest group containing $\{\lambda \mathrm{g}: \lambda$ is a real number and $\mathrm{g} \in \mathrm{V}\}$.

I require the convex regions to satisfy the following:

If regions $u$ and $g(u)$ are both parts of a convex region $w$ then so is $\mathrm{h}(\mathrm{u})$ where $\mathrm{h}=\lambda \mathrm{g}$ and $0<\lambda<1$.

I have taken convexity as primitive and required the symmetries to preserve it. I could have chosen some other shape that must be preserved by translation, provided it is sufficiently restrictive to ensure that there is a unique transitive commutative group of symmetries. If we took as fundamental the light cone structure or the associated ordering with respect to absolute, that is, frame-independent, priority, then it would suffice that there is a commutative group that acts faithfully, preserves cones and is transitive on cones, in the sense that given any two past light cones $\mathrm{u}$ and $\mathrm{v}$ some member of the group maps $\mathrm{u}$ to $\mathrm{v}$. To ensure that the regions labelled cones are the correct shape, it suffices that the group of all the symmetries leaving any given cone fixed is isomorphic to the group of those Lorentz transformations that do not reverse temporal orientation. This group may be characterised intrinsically. ${ }^{151}$

The requirement of a unique transitive commutative subgroup was relaxed in the analogous characterisation of a topological manifold, discussed previously, in which case the symmetries need only preserve the topological structure. The group of symmetries is then rather large but the requirement of being a patch for a topological manifold is that it contains some commutative subgroup acting transitively - it will in fact contain infinitely many.

\section{Generalising to the non-affine case}

In the point-based case we can generalise the symmetry-theoretic account rather easily. For the property of convexity does not require af-

151 It is generated by a connected group $G$ and a symmetry $j$, not in $G$, that is its own inverse, i.e. $\mathrm{j}^{2}=\mathrm{Id}$. $\mathrm{G}$ must be isomorphic to the connected component of the Lorentz group $\mathrm{SO}^{+}(1,3)$. This in turn is the unique 6 dimensional simple, connected, Lie group. 
fine structure. ${ }^{152}$ We are to suppose $\mathrm{G}$ is the group of all symmetries and that $\mathrm{G}$ acts transitively on the aether. But we no longer need them to act faithfully. Then consider any point part $\mathbf{o}$. We define an equivalence relation on $\mathrm{G}$ by saying that $\mathrm{g}$ and $\mathrm{h}$ are equivalent if they map $\mathbf{o}$ to the same point. Then the points are in one to one correspondence with the equivalence classes, the set of which is called the quotient space $\mathrm{G} / \mathrm{Stab}_{\mathbf{o}}$. If two symmetries act in the same way they must be equivalent. The correspondence correlates any point part $\mathrm{v}$ of the aether with the equivalence class of those $g$ sending $\mathbf{o}$ to $\mathrm{v}$. The point $\mathbf{o}$ plays the role of the origin in the correlation of an affine Space-time with a vector space. To be sure, a different choice of the origin, $\mathbf{o}^{\prime}$, would result in different equivalence classes, for in the non-commutative case we can have $\mathrm{g}(\mathbf{0})=$ $\mathrm{h}(\mathbf{0})$ but $\mathrm{g}\left(\mathbf{o}^{\prime}\right) \neq \mathrm{h}\left(\mathbf{o}^{\prime}\right)$. Nonetheless this procedure can be used to describe symmetric structure for the aether in terms of a group of symmetries $G$ and any subgroup $H$, which may be taken to be Stabo, for the origin $\mathbf{o .}^{153}$

Two examples illustrate this. The first is that in which the aether is circular in the three spatial dimensions, but still flat, in that it is locally indistinguishable from affine Space-time. In that case the group is commutative and I shall replace multiplicative by additive notation as is conventional. Consider then the commutative group V of symmetries that we recognise as the 4 dimensional vector space. And fix some point as the origin $\mathbf{0}$. Then if we represent $\mathrm{V}$ as the set of all coordinate quadruples, we may take $\mathrm{Stab}_{\mathbf{o}}$ to be $\mathrm{H}$, the set of all integer quadruples. $\mathrm{H}$

152 Each member of the Lie group, other than Id, generates either a line or a circle. In the line case we require the same condition on convexity as for the affine case. In the circle case we require that if $u$ and $g(u)$ are parts of a convex region $\mathrm{w}$, then so is $\mathrm{h}(\mathrm{u})$ for any $\mathrm{h}$ on the lesser of the two arcs connecting Id with $\mathrm{g}$. By the lesser arc I mean the one that is mapped into the other arc by inversion. In the special case in which both the arcs are lesser by this definition the definition of convexity requires that both be taken as the lesser arc.

We define the equivalence relation by saying that $g$ and $g^{\prime}$ are equivalent if for some $\mathrm{h} \in \mathrm{Hg}=\mathrm{g}^{\prime} \mathrm{oh}$. Then we take the points of the symmetric space to be represented by the equivalence classes. $\mathrm{H}$ is itself an equivalence class and represents the origin. 
may be characterised up to group isomorphism as the torsion-free commutative group with three generators but not two. ${ }^{154}$ The torsionfreedom is in fact redundant because $\mathrm{H}$ may be taken as any subgroup of $\mathrm{V}$ that has three generators but not two.

We would get the same result if we characterised a group of symmetries acting faithfully and with no fixed points (except for the identity symmetry which leaves every point fixed.) ${ }^{155}$ I prefer, however, to consider the whole of $\mathrm{V}$ as the group of symmetries on the grounds that the very same universal, a vector with a given length and direction, relates pairs of points in the affine case and the case in which some of the dimensions are curved up in a circle.

Another example is de Sitter Space-time. (I shall ignore the case of anti de Sitter Space-time: interested readers will be able to adapt what I say.) It is the result of replacing affine Space-time by a hyperspherical Space of radius that increases with Time. It may be represented by the set of quintuples $\left\{<\mathrm{v}, \mathrm{w}, \mathrm{x}, \mathrm{y}, \mathrm{z}>\right.$ : $\left.\mathrm{w}^{2}+\mathrm{x}^{2}+\mathrm{y}^{2}+\mathrm{z}^{2}=\mathrm{v}^{2}+\alpha^{2}\right\}$, where $\alpha$ is a constant. ${ }^{156}$ We may take the group of symmetries $G$, in this case acting faithfully on de Sitter Space-time, to be $\operatorname{SO}(1,4)$, that is those 5 by 5 matrices that act on the quintuples $<\mathrm{v}, \mathrm{w}, \mathrm{x}, \mathrm{y}, \mathrm{z}>$ preserving the quadratic expression $\mathrm{v}^{2}-\left(\mathrm{w}^{2}+\mathrm{x}^{2}+\mathrm{y}^{2}+\mathrm{z}^{2}\right)$ and which preserve orientation. If $\mathrm{u}$ is a point represented by $<\mathrm{v}, \mathrm{w}, \mathrm{x}, \mathrm{y}, \mathrm{z}>$, then $\mathrm{Stab}_{\mathrm{u}}$ is represented by those members of $G$ that leave fixed $<v, w, x, y, z>$. This is isomorphic to $\operatorname{SO}(1,3)$ the group of 4 by 4 matrices that preserve the quadratic expression $\mathrm{v}^{2}-\left(\mathrm{x}^{2}+\mathrm{y}^{2}+\mathrm{z}^{2}\right)$ and that preserve orientation.

154 By torsion-free we mean that for any positive integer $\mathrm{n}$ if $\mathrm{nx}=0$ then $\mathrm{x}=0$. To say $\mathrm{H}$ has $\mathrm{k}$ generators is to say that there are $\mathrm{k}$ members not included in any proper subgroup of $\mathrm{H}$.

155 The unique connected commutative Lie group of dimension 4 whose maximal compact subgroups are of dimension 3.

156 The de Sitter cosmology has the cosmological constant $\Lambda=3 / \alpha^{2}$. The expansion is exponential if time is measured as the proper time of a galactic cluster. 
Because there is an independent way of describing $\mathrm{SO}(1,4)$ and $\mathrm{SO}(1,3)$ then we have the required characterisation of de Sitter Space-time. ${ }^{157}$

If we compare the group of all symmetries for Sitter Space-time and for affine Space-time we see that the stabiliser of a given point in de Sitter Space-time is a smaller group than that for an affine Space-time of the same number of dimensions. So we may say that affine Space-time is more symmetric because it has an unrestricted spatio-temporal isotropy. In the de Sitter case the stabiliser is a Lorentz group and so isotropy is restricted in that we distinguish space-like, null, and time-like separations. As a consequence, matter-free de Sitter Space-time has a unique light cone structure, whereas affine Space-time has infinitely many.

Affine aether is initially somewhat more probable. First, absent other considerations it was preferable not to privilege the matter-free case as gravity free. (See the previous Chapter.) And second, I say, not merely is isotropy a priori probable, but the greater the isotropy the greater the probability. However, current cosmology supports de Sitter aether. This is because of the posited inflationary expansion soon after the Big Bang (Schmidt 1993). Such expansion is a consequence of de Sitter Space-time, in which case the subsequent deceleration of the expansion is explained as the result of matter. On the other hand if we suppose an affine aether we require something mysterious such as dark energy to explain the inflationary expansion.

$\mathrm{SO}(1,4)$ has a connected normal subgroup $\mathrm{SO}^{+}(1,4)$, such that the quotient is the group with just two members. $\mathrm{SO}^{+}(1,4)$ is one of only three Lie groups of 10 dimensions that is connected and 'simple' in the group theoretic sense of having no normal subgroup. A normal subgroup $\mathrm{N}$ of a group $\mathrm{G}$ is defined as one such that for all $g \in G$ and $h \in N, g-1 \circ h \circ g \in N$.

These three groups, $\mathrm{SO}^{+}(0,5), \mathrm{SO}^{+}(1,4)$, and $\mathrm{SO}^{+}(2,3)$ differ in the size of their maximal compact subgroup, being of 10 dimensions for $\mathrm{SO}^{+}(0$, $5)$, which is itself compact, 6 for $\mathrm{SO}^{+}(1,4)$ but only 4 for $\mathrm{SO}^{+}(2,3)$. Once we have picked out $\mathrm{SO}^{+}(1,4)$ as the unique Lie group of 10 dimensions that is connected, simple and has a compact subgroup of 6 dimensions but no more than 6 , we may note that stabiliser of any point $u$ is another 6 dimensional group whose maximal connected subgroup is the unique simple Lie group of 6 dimensions. 
As a consequence, the case for the symmetry-theoretic continuous aether over granulated aether is, I regret to say, weakened a little by Bayes' Rule in probability theory, because I 'predicted' affine aether but 'discovered' de Sitter aether.

There is another, happier, consequence. Because the light cone structure is specified in de Sitter aether, we might as well use light cones rather than convexity as the structure that is to be preserved by the symmetries and this will turn out to save the point-free de Sitter case from an objection to be discussed below.

\section{Spatio-temporally restricted aether}

Those of us who hold a dynamic theory of Time should deny the reality of any future aether. Nonetheless, as I shall argue, we can believe in future Space-time. Maybe some of those who hold that the universe came into existence a finite time ago might likewise hold that Space-time predated the aether. Perhaps some presentists hold that the aether is nothing but a thin layer, a Planck time thick, say. They too might believe in Space-time that has an infinite past and future.

In all these cases we may use the group-theoretic approach to construct Space-time from a partially symmetric aether if three conditions hold. The first is that the aether is connected. The second is that it is the sum of open convex regions. (This excludes the case in which the physical universe is made up of point-particles, spatially one dimensional strings, or indeed branes of spatial dimension less than that of Space itself.)

The third condition is that of determinate spatial extent. Its most straightforward version is that in which the aether is infinite in all spatial dimensions. The less straightforward case is that in which there is an upper bound to how far the objects are away but that is because Space is toroidal so as we go farther away on a certain direction we eventually come back to where we start. ${ }^{158}$ The condition is that all such questions should be settled. So it excludes the case of a finite universe that could equally well be described as occupying part of an infinite Space or a fi-

There are other, less plausible, cases, namely those in which Space is finite in some directions, but infinite in others, like the surface of a cylinder. 
nite, toroidal Space. It does not, however, exclude the presentist hypothesis that the aether is a slice of finite temporal thickness because I hold that circular Time is impossible.

We could try using the theory of partially symmetric stuff to provide an alternative to an aether pervading the whole universes. For suppose there are extended particles composed of some sort of stuff, which I shall call prime matter. Then we hypothesise that the prime matter occupies an open part of Space-time. And suppose the particles undergo fission and fusion. Then the prime matter constituting two particles that fuse makes up a connected open region. Likewise for two particles arrived at by fission. Given enough fission and fusion it is plausible that the sum of the prime matter would be spatio-temporally connected. And we may assume it is the sum of open convex regions. So in this case Space-time may be constructed out of the prime matter rather than an all-pervading aether. The same would hold if there was not fission and fusion but particles that collided with distortion so that they came into contact along surface rather than just at a point or just along an edge. ${ }^{159}$ My reason for rejecting this way of avoiding the all-pervading aether is that the aether's existence is non-contingent and hence no doubt is cast upon it by a thoroughly contingent arrangement of particles.

A group of partial symmetries may be defined by relaxing some of the conditions required for a group of symmetries, considered as dyadic relations. The relations still correspond to one to one functions, that is, they are relations $\operatorname{Rg}$ such that (1) if $\operatorname{Rgxy}$ and $\operatorname{Rgxz}$ then $y=z$, and (2) If Rgwy and $\operatorname{Rgxy}$ then $w=x$. But we no longer require that for every point $\mathrm{x}$ there is some $\mathrm{y}$ such that $\mathrm{Rgxy}$. The identity relation Id relates every region to itself and we still require that for any $g$ in the group there is an inverse g-1 its converse. (So if S is the inverse of R, Sxy if and only if Ryx.)

As far as I can see, contact in just one or two dimensions would permit convexity-preserving symmetries that rotate one particle relative to another, which by enlarging the group of (partial) symmetries would interfere with the symmetry-theoretic characterisation. 
I note that the composition of members of the group no longer corresponds to the product of relations. For if Rgxy and Rhyz then, to be sure, Rgohxz, but the converse need not hold. I shall also assume that the group acts with local fidelity. That is if for some open region $\mathrm{u} g(\mathrm{x})$ and $\mathrm{h}(\mathrm{x})$ are 'defined' and identical for every part of $\mathrm{u}$, then $\mathrm{g}=\mathrm{h} .{ }^{160} \mathrm{Be}-$ cause the aether is open and connected this shows that the symmetries are maximal in that there are no two symmetries $g$ and $h$ such that $h$ is the restriction of $g$ to some proper sub-domain. That is, we cannot have $\mathrm{g} \neq \mathrm{h}$ such that $\mathrm{Rgxy}$ only if $\mathrm{Rhxy}$.

A group $G$ of partial symmetries can be used to construct Spacetime as follows. Given any point parts $u$ and $x$ we can consider those partial symmetries $\mathrm{g}$ such that $\mathrm{g}(\mathrm{u})=\mathrm{x}$, including the special case in which $\mathrm{u}=\mathrm{x}$ and $\mathrm{g}=\mathrm{Id}$. In the previous section I considered the case of an all-pervading point-based aether. In that case we can, if we wish, identify Space-time with the aether. But I now consider how we may use the group of partial symmetries to construct the Space-time locations in such a way that every region of the aether has for its locations every member of the corresponding set of properties.

Given any point parts $u$ and $x$ we can consider those partial symmetries $\mathrm{g}$ such that $\mathrm{g}(\mathrm{u})=\mathrm{x}$. If $\mathrm{g}$ is such a partial symmetry, $\mathrm{x}$ has the relational property $\mathrm{Lg}$; $\mathrm{u}$ of having a part standing in the converse of $\mathrm{Rg}$ to $\mathrm{u}$. If there is no point-part of the aether $\mathrm{x}$ such that this holds then the relational property is uninstantiated. I shall permit this but I still require $u$ to exist for there to be a genuine property $\mathrm{Lg}$; $\mathrm{u}$. We may define an equivalence relation on the set $\mathrm{LG}$ of those $\mathrm{Lg} ; \mathrm{u}$ for which $\mathrm{g}$ is in $\mathrm{G}$ and $\mathrm{u}$ is a point-part of the aether by: $\mathrm{Lg}_{\mathrm{g}} \mathrm{u} \sim \mathrm{Lk} ; \mathrm{v}$ just in case $\mathrm{h} \circ \mathrm{g}(\mathrm{u})=\mathrm{h} \circ \mathrm{k}(\mathrm{v})$ for some partial symmetry $h$. And we may take a location to be the conjunction of all the members of $\mathrm{LG}_{\mathrm{G}}$ in a given equivalence class. So every point-part of the aether instantiates a location and Space-time is composed of all the locations, instantiated or otherwise.

This way of constructing Space-time using uninstantiated properties requires that the aether characterises uniquely the group of partial 
symmetries G. The theory of Lie groups shows that the way the group acts on any open region $\mathrm{V}$ already significantly constrains the group. Taken together with the conditions stated above, this show there is a unique connected Lie group that acts as partial symmetries on the aether. For example, in the affine case the fact that $\mathrm{G}$ has a subgroup $\mathrm{H}$ acting on $\mathrm{v}$ in a transitive commutative fashion shows that $\mathrm{H}$ is commutative. The existence of parts of the aether arbitrarily far away in each spatial direction together with the assumption that Time is non-circular shows that $\mathrm{H}$ is isomorphic to the a four dimensional vector space. Every member of $\mathrm{LG}_{\mathrm{G}}$ is equivalent to some member of $\mathrm{LH}$, so the set of locations have the required affine structure.

\section{Symmetric point-free aether.}

To provide a symmetry-theoretic account of point-free aether, such as the favoured hypothesis of Arntzenius Continuum, I adopt the following procedure: (1) Modify the point-based characterisation so that it no longer mentions points; and (2) show how a point-based Space-time may be constructed from the point-free aether. First I begin with a general method that relies on the aether having a light cone structure, or equivalently a suitable partial ordering of absolute (frame-independent) priority, that specifies gravity in the matter-free case. In the last chapter I submitted that it was preferable not to posit such a light cone structure, and in general it was fairly easy to avoid this, using convexity instead. However, de Sitter and anti de Sitter Space-time come equipped with light cones. So this method is appropriate for those structures. Currently it seems quite likely that mass-free Space-time is a de Sitter Space-time, but the a priori preferable case of affine aether should not be ignored. Therefore I also need to discuss an alternative characterisation for the flat case, including affine aether.

Because cones are pointy and because there is only one past light cone through each point, we may use the past light cones as surrogates for points. My reason for preferring past light cones over future ones is that if there is past aether but no future aether I would like the (constructed) locations of the most recent light cones to include the present. I could just as easily have considered double cones. 
The symmetries must preserve the mereological structure, the light cones, and hypervolume, as well as preserving the symmetries themselves. The action of a group of symmetries is faithful if no two symmetries act on regions in the same way and transitive if for any two future light cones, $u$ and $v$ there is some symmetry g such that $g(u)=v$. So the first part of the procedure is easy.

The construction of Space-time is just as straightforward. The Alexandrov intervals are defined as the non-empty intersections of past and future light cones. If $\mathrm{w}$ is any past light cone, I say an Alexandrov interval $\mathrm{x}$ fits $\mathrm{w}$ if there is no Alexandrov interval $\mathrm{y}$ that is also part of $\mathrm{w}$ such that $\mathrm{y}$ is disjoint from some past light cone $\mathrm{v}$ that $\mathrm{x}$ is also part of. Then given any past light cone $\mathrm{v}$ and symmetry $\mathrm{g}$ we may consider the property $\mathrm{Mg}$; $\mathrm{v}$ of intersecting every Alexandrov interval that fits $\mathrm{g}(\mathrm{v})$. Let $\mathrm{MG}_{\mathrm{G}}$ be the set of all such $\mathrm{Mg}_{\mathrm{v}} \mathrm{v}$ and define an equivalence relation on $\mathrm{MG}_{\mathrm{G}}$ by $\mathrm{Mg} ; \mathrm{u} \sim \mathrm{M}_{\mathrm{k} ; \mathrm{v}}$ if $\mathrm{h} \circ \mathrm{g}(\mathrm{u})=\mathrm{h} \circ \mathrm{k}(\mathrm{v})$ for some symmetry $\mathrm{h}$. The conjunction of members of an equivalence class (instantiated or otherwise) is then a point location. Every part of the aether may be represented as the set of all locations belonging to it.

This way of characterising the shape of the aether, and of constructing points, requires that there be enough symmetries mapping past cones to past cones, and so is not available if there is a thin present layer of aether - or, on the, already rejected, hypothesis of extended particles made of prime matter. We can, however, modify the hypothesis by requiring not that past light cones be preserved but that Alexandrov intervals (and of course hypervolume) be. This will give us enough partial symmetries and we may then characterise transitivity by saying that given any two Alexandrov intervals of the same hypervolume some partial symmetry maps one to the other.

The cone-free, point-free affine case has one annoying complication, namely the difficulty of characterising a suitable analog for transitivity. The problem is that in the point-free case we require that for any regions $u$ and $v$ of the same shape and with the same size and situated in a parallel way, some translation (vector) maps $u$ to v. A structure of light cones (and hence of Alexandrov intervals) provided us with a suitable conception of being the same shape, and all future light cones are point- 
ing in the same direction, but things are more complicated if convex regions are used in place of light cones. We can characterise a half as a convex region whose complement is convex. In the Arntzenius Continuum case all halves are the same shape but they are not all arranged in a parallel way. Hence we require rotations as well as translations to map any half to any half. We can characterise transitivity by requiring that given any halves $\mathrm{u}$ and $\mathrm{v}$, if $\mathrm{u}$ and $\mathrm{v}$ have parallel boundaries, and so 'point' in the same direction, some symmetry maps $u$ to $v$. If the aether extends infinitely in all directions, including the future, this parallel boundary condition may itself be analysed as one half being part of the other.

A problem arises, however, for those who hold that there is no future aether. For then two hyperplanes in Space-time that intersect in the future will turn out to bound halves of the aether one of which is part of the other. Initially, therefore, we might infer that the combination of belief in affine Space-time and no future aether excludes Arntzenius Continuum. Instead of drawing this conclusion we could adapt the characterisation of topological manifold in the point-free case, described in Chapter Four. But it gets messy. It is neater to treat being parallel as primitive, but that is uneconomic.

The point locations may be constructed using ultrafilters. ${ }^{161}$ In the case in which the aether does not occupy the whole of Space-time, con-

161 We might also construct Space-time by noting that each point $\mathrm{p}$ of Spacetime sets up a way of assigning subsets of the group $\mathrm{V}$ of vectors to regions, namely the vectors that map $p$ to points in the region. In the case of allpervading aether, we may use this to characterise a point as a map $\psi$ from non-empty convex open sets of vectors to convex open regions such that:

1. If $U$ is any non-empty set of non-empty open convex sets of vectors whose union $\mathrm{u}$ is convex, then $\psi(\mathrm{u})$ is the least upper bound of $\{\psi(\mathrm{x})$ : $\mathrm{x} \in \mathrm{U} \underline{\underline{y}}$.

2. $\psi(\mathrm{X} \cap \mathrm{Y})=\psi(\mathrm{X}) \wedge \psi(\mathrm{Y})$ if $\mathrm{X} \cap \mathrm{Y} \neq \varnothing ; \psi(\mathrm{X})$ and $\psi(\mathrm{Y})$ are disjoint if $\mathrm{X} \cap \mathrm{Y}=\varnothing$

3. If $\mathrm{v}$ is any vector, $\psi(\{\mathrm{v}+\mathrm{x}: \mathrm{x} \in \mathrm{X}\})=\mathrm{v}(\psi(\mathrm{X}))$.

A mapping $\psi$ may then be said to be a location of a region $\mathrm{u}$ if every open convex set of vectors that contains the identity (zero) vector is mapped by $\psi$ into some region that overlaps $u$. This construction is, however, more com- 
sider the property $\mathrm{Ng}_{\mathrm{g}} \mathrm{v}$ that a region of aether has/would have if inter-

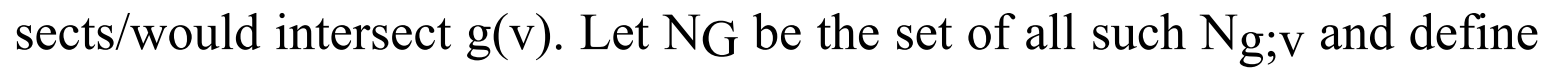
a transitive anti-symmetric relation on $\mathrm{NG}_{\mathrm{G}}$ by $\mathrm{Ng}_{\mathrm{g}} \mathrm{u}$ « $\mathrm{Nk}$; $\mathrm{if}$ h०g(u) is an interior part of $\mathrm{h} \circ \mathrm{k}(\mathrm{v})$ for some $\mathrm{h}$. Then a point location is the conjunction of all the properties in $\mathrm{NG}_{\mathrm{G}}$ that belong to a given ultrafilter with respect to «. This is a large conjunction of fairly straightforward relational properties, which I judge to be a satisfactory structure for a nonfundamental property to have.

\section{Extending symmetry to many 'worlds'}

In the Introduction, I noted that we might hold that there are many worlds but that I would concentrate on just one of them. That is justified provided we are considering many spatio-temporally disconnected worlds, as in Lewis' theory. In that case it is strictly speaking false to say that the aether has, for instance, affine structure. But the required modification of hypotheses is obvious - each connected component has, in this case, affine structure.

Instead, we might well hold that that actual Universe is composed of many spatio-temporally connected universes. Either they are now connected (with respect to any frames of reference) or, if no longer connected, they form a branching structure, where in a branching Universe, given any two regions $u$ and $v$ there is some region that absolutely prior to them both (McCall 1994). If we hold some such 'many worlds' theory, perhaps as an interpretation of quantum theory, then we should examine the implications for the investigation of the structure of the aether.

The branching Universe is the sum of overlapping universes. Given any two such universes $\mathrm{x}$ and $\mathrm{y}$ their meet $\mathrm{x} \wedge \mathrm{y}$ is a like an non-branching universe up to some Space-like hypersurface. If the branching is ordered by the integers then this may be taken as the discrete Time mentioned in Chapter Six, which I argued was compatible with a continuous aether. If we are realists about both the future and the past aether then each of the universes may be characterised using symmetry. They might, for in-

plicated than resort to ultrafilters when interpreted in terms of an ontology of properties and relations. 
stance, all have affine structure or all have de Sitter Space-time structure. The only implication seems to be that a branching Universe supports the distinction of Time from Space-time and so offers a reply to the objection, that some might find initially persuasive, that this distinction is an ad hoc way of saving continuous theories from arguments for discrete Time.

If the many universes are always connected, then they may be interpreted as being located in 4 dimensional fibres that an $\mathrm{N}+1$ dimensional Space-time is divided into, where $\mathrm{N}$, the number of spatial dimensions, is greater than 3. (I use the term 'fibres' even though they have 3 spatial dimensions because this is standard mathematical terminology and the higher dimensional Space-time would be said to have a fibration. See Rowland, 2009 ) We, the observers, may then be thought of as having more than 3 spatial dimensions, with the apparent indeterminacy of the physical universe being due to the very slight differences between the states of the fibres we overlap. Given the case for Arntzenius Continuum the 4 dimensional universes are themselves constructs, like points because every region is of more than 4 dimensions. But that's fine.

The $\mathrm{N}+1$ dimensional aether could have the structure of a higher dimensional Minkowski Space-time even though the fibres are de Sitter Space-times. For simplicity consider $N=4$. Then for any $\mathrm{k}>0$, the hypersurface $\left\{<\mathrm{v}, \mathrm{w}, \mathrm{x}, \mathrm{y}, \mathrm{z}>\right.$ : $\left.\mathrm{w}^{2}+\mathrm{x}^{2}+\mathrm{y}^{2}+\mathrm{z}^{2}-\mathrm{v}^{2}=\mathrm{k}\right\}$ represents a de Sitter Space-time. By varying the $\mathrm{k}$ we can stack the hypersurfaces up neatly to obtain a fibration of the region of a 5 dimensional Minkowski aether represented by $\left\{<\mathrm{v}, \mathrm{w}, \mathrm{x}, \mathrm{y}, \mathrm{z}>: \mathrm{w}^{2}+\mathrm{x}^{2}+\mathrm{y}^{2}+\mathrm{z}^{2}-\mathrm{v}^{2}>0\right\}$.

The higher dimensional Minkowskian aether may easily be characterised using symmetries, using the pointy nature of the light cones. But there is a further implication: I do not know how to characterise de Sitter aether axiomatically, but the Minkowskian case is amenable to an axiomatic treatment. ${ }^{162}$ This overcomes an obstacle in the way of those nom-

162 The difficulty with an axiomatic characterisation of de Sitter spaces is that spatially they are like spherical geometry but temporally they are like a hyperbolic geometry. So nominalist might need to distinguish Space from Time to overcome this difficulty. 
inalists who would otherwise accept symmetric Arntzenius Continuum, but who reject the group-theoretic characterisation of symmetric aether, and who take de Sitter Space-time to be more probable than Minkowski Space-timefor the non-quantum 4 dimensional case.

If some or all of the 'worlds' are of infinite extent, either spatially or temporally, then we might require an infinite dimensional Minkowskian Space-time to contain them all. 